\title{
Fatal cerebral infarct in a child with COVID-19
}

\section{Shingo Kihira ${ }^{1}$ (I) $\cdot$ Peter F. Morgenstern ${ }^{2} \cdot$ Hillary Raynes $^{3} \cdot$ Thomas P. Naidich $^{1} \cdot$ Puneet Belani $^{1}$}

Received: 1 June 2020 / Revised: 17 June 2020 / Accepted: 8 July 2020 / Published online: 23 July 2020

(C) Springer-Verlag GmbH Germany, part of Springer Nature 2020

\section{Dear Editors,}

Severe acute respiratory syndrome coronavirus-2 (SARS$\mathrm{CoV}-2$ ) causes a cytokine storm through angiotensinconverting enzyme 2 (ACE2) receptor binding, leading to a hypercoagulable state $[1,2]$. This has led to increased incidence of vascular thromboses in people with coronavirus disease 2019 (COVID-19) [3, 4]. It has been reported that children with SARS-CoV-2 infrequently have severe disease [5]. At our institution in New York City, we saw only four cases of children presenting with SARS-CoV-2 infection and demonstrating Kawasaki-like symptomatology between March 15 and April 30 of 2020. Here we write about an unusual case to raise awareness that a post-infectious syndrome associated with SARS-CoV-2 can be fatal in children.

A 5-year-old boy with no significant medical history presented with 3 days of fever, cough and abdominal pain. He was diagnosed with prior COVID-19 infection through plasma antibody assay. D-dimer at presentation was elevated $(12 \mu \mathrm{g} / \mathrm{mL}$; normal range $<0.50 \mu \mathrm{g} / \mathrm{mL})$. He was transferred to our hospital in New York City and started on a heparin drip. He became hypotensive and was moved to the pediatric intensive care unit, where he was intubated and started on titrated epinephrine infusion. Chest radiograph demonstrated coarse bronchovascular prominence and mild cardiomegaly. An echocardiogram demonstrated an ejection fraction of $30 \%$ and no structural cardiac anomalies. Cardiogenic shock secondary to inflammatory cytokine storm was suspected

Shingo Kihira

shingokihira@gmail.com

1 Department of Diagnostic, Molecular and Interventional Radiology, Icahn School of Medicine at Mount Sinai,

One Gustave L. Levy Place, Box 1234, New York, NY 10029, USA

2 Department of Neurosurgery,

Icahn School of Medicine at Mount Sinai, New York, NY, USA

3 Department of Pediatrics,

Icahn School of Medicine at Mount Sinai, New York, NY, USA because of recent COVID-19 infection. He underwent venoarterial extracorporeal membrane oxygenation (ECMO) cannulation. Over the next 4 days his condition improved. Hours prior to decannulation, his right pupil became dilated and nonreactive to light. Emergent decannulation was performed and a head CT (Fig. 1) revealed a large acute right anterior and middle cerebral artery territory infarction and subarachnoid hemorrhage in the left hemisphere. Immediately following CT scan his left pupil became fixed and dilated. Sugammadex was administered for rapid reversal of his residual vecuronium paralysis. Neurologic exam demonstrated

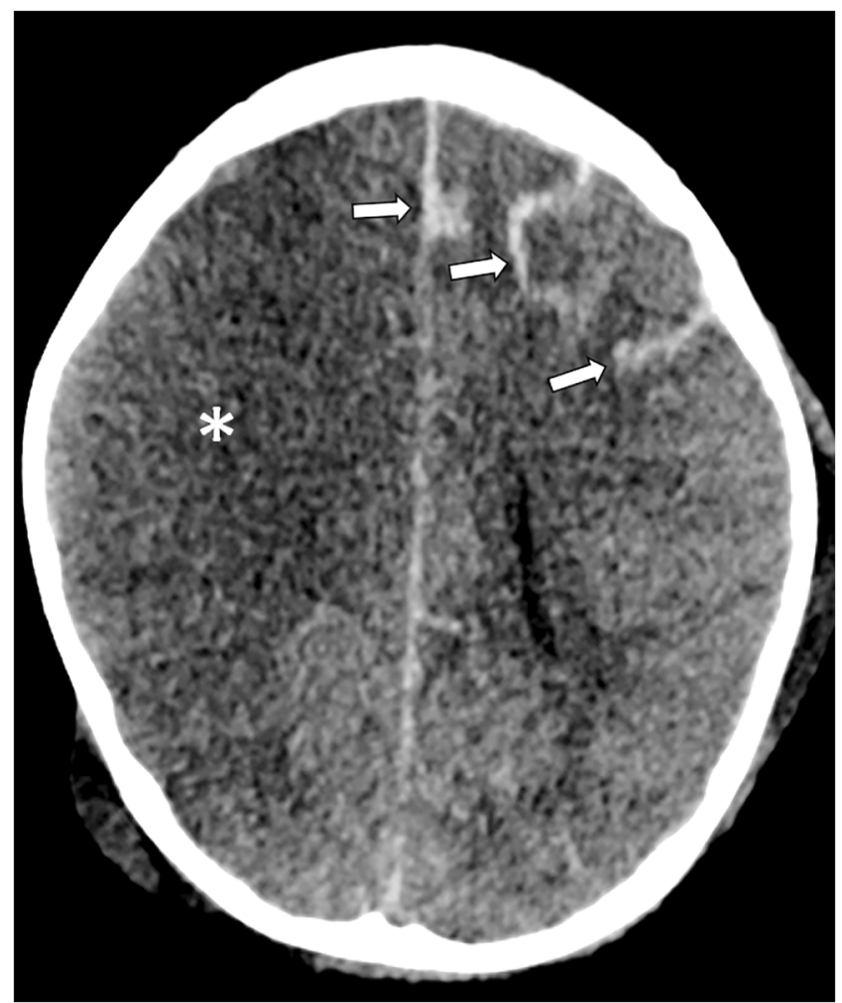

Fig. 1 Axial non-contrast head CT in a 5-year-old boy with recent COVID-19 infection demonstrates massive right anterior and middle cerebral artery infarction (asterisk) and subarachnoid hemorrhage in the left hemisphere (arrows) 
absent corneal, gag and pupillary responses and no movement to painful stimuli. After discussion with his family no surgical decompression was attempted because of his clinical condition. He was declared brain dead 3 days later.

This is an unfortunate case of a healthy 5-year-old boy with COVID-19 infection complicated by cardiogenic shock and hypercoagulable state. The combination of cardiogenic shock and hypercoagulable state presumably contributed to the massive cerebral infarction, with the contributing role of ECMO cannulation uncertain. It is our hope that this case informs caregivers and imagers of a potentially devastating neurologic manifestation of COVID-19.

\section{Compliance with ethical standards}

Conflicts of interest None

\section{References}

1. Mehta P, McAuley DF, Brown M et al (2020) COVID-19: consider cytokine storm syndromes and immunosuppression. Lancet 395: 1033-1034

2. Connors JM, Levy JH (2020) COVID-19 and its implications for thrombosis and anticoagulation. Blood 135:2033-2040

3. Panigada M, Bottino N, Tagliabue $P$ et al (2020) Hypercoagulability of COVID-19 patients in intensive care unit. A report of thromboelastography findings and other parameters of hemostasis. J Thromb Haemost. https://doi.org/10.1111/jth.14850

4. Zhang Y, Xiao M, Zhang S et al (2020) Coagulopathy and antiphospholipid antibodies in patients with Covid-19. N Engl J Med 382:e38

5. Kelvin AA, Halperin S (2020) COVID-19 in children: the link in the transmission chain. Lancet Infect Dis 20:633-634

Publisher's note Springer Nature remains neutral with regard to jurisdictional claims in published maps and institutional affiliations. 